\title{
Presented antigen from damaged pancreatic $\beta$ cells activates autoreactive $T$ cells in virus-mediated autoimmune diabetes
}

\author{
Marc S. Horwitz, Alex Ilic, Cody Fine, Enrique Rodriguez, and Nora Sarvetnick \\ Department of Immunology, The Scripps Research Institute, La Jolla, California, USA \\ Address correspondence to: Nora Sarvetnick, Department of Immunology (IMM-23), The Scripps Research Institute, \\ 10550 North Torrey Pines Road, La Jolla, California 92037, USA. \\ Phone: (858) 784-9016; Fax: (858) 784-9096; E-mail: noras@scripps.edu.
}

Received for publication August 30, 2000, and accepted in revised form October 29, 2001.

\begin{abstract}
The induction of autoimmunity by viruses has been attributed to numerous mechanisms. In mice, coxsackievirus B4 (CB4) induces insulin-dependent diabetes mellitus (IDDM) resembling the final step of disease progression in humans. The immune response following the viral insult clearly precipitates IDDM. However, the molecular pathway between viral infection and the subsequent activation of $T$ cells specific for islet antigen has not been elucidated. These $T$ cells could become activated through exposure to sequestered antigens released by damaged $\beta$ cells, or they could have responded to factors secreted by the inflammatory response itself. To distinguish between these possibilities, we treated mice harboring a diabetogenic $\mathrm{T}$ cell repertoire with either the islet-damaging agent streptozotocin (STZ) or poly I:C, which nonspecifically activates T cells. Significantly, only treatment of mice with STZ resulted in IDDM and mimicked the effects observed following CB4 infection. Furthermore, antigen-presenting cells from STZ-treated mice were shown to directly activate autoreactive $\mathrm{T}$ cells and induce diabetes. Therefore, the primary role of CB4 in the precipitation of IDDM is to damage tissue, causing release and presentation of sequestered islet antigen. These events stimulate autoreactive $T$ cells and thereby initiate disease.
\end{abstract}

J. Clin. Invest. 109:79-87 (2002). DOI:10.1172/JCI200211198.

\section{Introduction}

Coxsackieviruses have long been associated with the development of insulin-dependent diabetes mellitus (IDDM) (1-4). Understanding how coxsackieviruses mediate diabetes has been complicated by this virus's unique tropism for the pancreas as well as the apparent molecular similarity between the viral P2C gene product of the B4 strain (CB4) and the pancreatic islet antigen GAD65 (5). Recently, the mechanistic role of this virus was dissected by infecting mice representing different experimental models of diabetes (6), and the results favored a mechanism of $\mathrm{T}$ cell activation through bystander damage over induction through molecular mimicry. CB4 infection of NOD mice, which carry the NOD MHC allele to which presentation of the cross-reactive epitope is restricted, did not enhance or change the onset or progression of diabetes. Additionally, no proliferative response to either of the crossreactive epitopes of $\mathrm{P} 2 \mathrm{C}$ or GAD was observed following infection. However, CB4 infection of BDC2.5 transgenic $(\mathrm{Tg})$ mice, which harbor a transgene encoding a diabetogenic $T$ cell receptor (TCR) specific to an islet granule antigen other than GAD65 (7) and not cross-reactive with $\mathrm{CB} 4$, resulted in the rapid onset of diabetes (6). In our colony, BDC2.5 Tg mice develop peri-insulitis at an accelerated rate but do not progress to spontaneous diabetes. CB4 infection activated the $\mathrm{Tg}$ memory $\mathrm{T}$ cell population prior to disease onset, precipitating clinical diabetes in these mice.
Whether memory $\mathrm{T}$ cells can be activated due to the release of sequestered antigen from the damaged tissue or simply by cytokine induction following viral infection remained as an important question. To address this possibility, BDC mice were infected with lymphocytic choriomeningitis virus (LCMV), which can crossactivate nonspecific resting memory lymphocytes during infection but does not destroy islet cells. LCMV infection did not induce diabetes nor any increase in insulitis (6). Therefore, viral expansion of nonspecific $\mathrm{T}$ cell responses through cytokine induction is most likely not activating islet-specific memory $\mathrm{T}$ cells to mediate disease. Yet, the dynamics of the LCMV infection may somehow suppress $(8,9)$ or interfere with (10-12) disease development.

To more completely distinguish between the mechanisms that account for coxsackievirus-mediated IDDM, we treated BDC2.5 mice with two environmental agents that mimic aspects of viral infection. Treatment with poly I:C effectively induces type 1 cytokine activation similar to that observed following viral infection (13). Alternatively, administration of a single subdiabetogenic dose of streptozotocin (STZ) directly damages $\beta$ cells in the pancreatic islets of Langerhans with only minimal immune cell involvement (14-17). If coxsackievirus induces IDDM through damage to $\beta$ cells, antigen release, and activation of self-reactive $T$ cells, then treatment of BDC2.5 Tg mice with STZ and not poly I:C should activate the resting anti-islet memory $\mathrm{T}$ cells, 
leading to the rapid development of diabetes. If, however, viral infection merely activates the resting autoreactive $\mathrm{T}$ cells via a cytokine response, then poly I:C treatment alone should be sufficient to induce disease.

\section{Methods}

Mice and virus. NOD/Shi and NODSCID mice were obtained from the rodent breeding colony at The Scripps Research Institute. BDC2.5 Tg mice were a gift from Diane Mathis (Joslin Diabetes Center, Harvard University, Boston, Massachusetts, USA; formerly of Institut de Genetique et de Biologie Moleculaire, Strassbourg, France) and have been maintained and bred to NOD/Shi in our mouse colony. Virus stocks of coxsackievirus group B type 4 Edwards strain 2 (CB4 strain E2) were obtained from Charles Gauntt (U. of Texas-San Antonio, San Antonio, Texas, USA) and were derived from stocks obtained from Roger Loria (Medical College of Virginia, Commonwealth University of Virginia, Richmond, Virginia, USA; ref. 3). CB4 was originally isolated from patients with IDDM, passaged through murine pancreatic $\beta$ cells, and subsequently found to induce a diabetes-like disease in mice $(3,4)$. Virus stocks of CB4 strain E2 were prepared and titered as previously described (18). Mice were infected at 12 weeks of age intraperitoneally with 100 plaqueforming units ( $\mathrm{pfu}$ ) of CB4. Mice were treated with 100 $\mu \mathrm{g}$ per mouse of poly I:C (Sigma Chemical Co., St. Louis, Missouri, USA) in PBS with an intraperitoneal injection. Mice were treated with STZ (Sigma Chemical Co.) at various concentrations as indicated in the text. STZ was prepared in citrate buffer immediately prior to intraperitoneal injection. Blood glucose was measured in tail vein or eye bleeds from nonfasting mice at various times from 1-28 days postinfection or posttreatment on a standard glucometer with a range of $20-400 \mathrm{mg} / \mathrm{dl}$. Mice were considered diabetic when two or more consecutive readings ( 24 hours apart) were greater than $300 \mathrm{mg} / \mathrm{d}$.

Immunohistochemical staining. Organs were removed and placed in $10 \%$ formaldehyde, then processed for paraffin sectioning. Alternatively, organs were snapfrozen in OCT on dry ice. Immunohistochemistry was performed on $4-\mu \mathrm{m}$-thick paraffin sections for insulin staining or $7-\mu \mathrm{m}$ cryocut sections for the other antibodies that were prepared and blocked with avidin and biotin (Vector Laboratories Inc., Burlingame, California, USA). The primary antibodies were to insulin (DAKO Corp., Carpinteria, California, USA), F4/80 (Serotec Inc., Washington, DC, USA), CD4 and CD25 (PharMingen, La Jolla, California, USA), and the secondary antibody was a biotinylated anti-guinea pig $\operatorname{IgG}$ (for insulin staining) or anti-rat IgG (for the other antibodies) used in conjunction with Vectastain ABC (peroxidase) kits (Vector Laboratories Inc., Carpinteria, CA). Staining was detected using diaminobenzidine as a chromagen. Sections were counterstained in Mayer's hematoxylin (Sigma Chemical Co.) and mounted in Permount (Fisher Scientific Co., Pittsburgh, Pennsyl- vania, USA). Quantitation of the degree of pancreatic insulitis was evaluated using both anti-insulin-stained and multiple hematoxylin and eosin-stained pancreatic sections from infected and uninfected, age-matched NOD and BDC2.5 Tg mice, and the mice were scored for peri-insulitis and insulitis. Statistical analysis using $\chi$-square tests were done on paired groups.

FACS analysis. Peripheral blood was obtained from mice before and 1 week after treatment with STZ or poly I:C. Erythrocytes were lyzed by $\mathrm{NH}_{4} \mathrm{Cl}$ treatment and lymphocytes incubated with phycoerythrin-labeled (PE-labeled) antibody to the TCR variable chain $V_{\beta} 4$ and Cy-chrome-labeled CD4 (PharMingen), and FITClabeled antibody to CD25 (Sigma Chemical Co.), CD44, or CD69 (PharMingen). Cells were fixed in 1\% paraformaldehyde and analyzed with a Becton Dickinson FACS. A minimum of 200,000 events were analyzed for each sample.

Proliferation assays. Antigen-presenting cells (APCs) were enriched from the spleens of untreated and STZtreated NOD and NODSCID mice by plastic adherence as previously described (19). Briefly, spleens were harvested and forced through mesh screens to generate single cell suspensions. Erythrocytes were lysed by $\mathrm{NH}_{4} \mathrm{Cl}$ treatment, and the remaining cells were resuspended in RPMI with $10 \%$ FCS at 10 million cells per milliliter. Cells were incubated on Petri dishes for 3 hours $\left(37^{\circ} \mathrm{C}\right.$, $\left.5 \% \mathrm{CO}_{2}\right)$. Afterwards, nonadherent cells were removed by multiple washes with RPMI. Adherent cells were removed by scraping, counted, and placed in appropriate media for use in either $\mathrm{T}$ cell proliferation assays or adoptive transfer. For proliferation assays, the APCenriched population was irradiated (15 Gy).

For $T$ cell proliferation assays, spleens from untreated BDC2.5 TCR Tg mice were harvested, and erythrocytes were removed and cultured in triplicate on 96well plates at $2 \times 10^{5}$ cells per well in HL- 1 media. To the BDC splenocytes, in triplicate, varying amounts of adherent cells were added $\left(0,1.25 \times 10^{4}, 2.5 \times 10^{4}\right.$, $5 \times 10^{4}$, or $10 \times 10^{4}$ cells). Additionally, triplicate controls with added APCs and peptide $(20)(7 \mu \mathrm{M})$ were performed. Cultures were incubated for 2 days at $37^{\circ} \mathrm{C}$ and pulsed for 18 hours before harvest with $1 \mu \mathrm{Ci}$ ${ }^{3} \mathrm{H}$-thymidine. Incorporated ${ }^{3} \mathrm{H}$ was measured using a liquid scintillation counter. The stimulation index was calculated for each splenocyte/APC sample, as compared with control wells incubated on the same plate but lacking any additional adherent cells or antigen.

Adoptive transfer. Donors were either 12-week-old untreated BDC2.5 TCR Tg mice or 6- to 8-week-old untreated or STZ-treated NODSCID mice. From BDC $\mathrm{Tg}$ mice, splenocytes were harvested, single cell suspensions generated, and erythrocytes removed, counted, and transferred at $2 \times 10^{6}$ to $5 \times 10^{6}$ cells per mouse. Recipient mice for the BDC splenocytes were NODSCID mice that had been treated with either STZ or PBS 7 days before. Alternatively, splenocytes from treated NODSCID mice were harvested and enriched for APCs by adherence to plastic as described above. APCs were trans- 
ferred into untreated 12 -week-old BDC mice at $5 \times 10^{5}$ to $10 \times 10^{5}$ cells per mouse. Mice were monitored weekly for changes in blood glucose.

\section{Results and Discussion}

Prior work has established the ability of poly I:C, a double-stranded nucleic acid, to invoke a type 1 IFN antiviral response without the accompanying damage of a viral infection (21). Additional work by Tough et al. (13) had shown that poly I:C treatment could act similarly to LCMV infection and stimulate nonspecific memory $\mathrm{T}$ cells. Whether these activated $\mathrm{T}$ cells could then participate in an autoimmune or pathologic response has been controversial. To examine whether the upregulation of the type 1 IFN pathway could induce IDDM through stimulation of memory T cells, we treated BDC2.5 Tg mice with poly I:C (100 $\mu \mathrm{g}$ per mouse) and monitored for disease onset. While CB4 induces diabetes in $67 \%$ of the infected BDC2.5 Tg mice within 4 weeks, poly I:C treatment was unable to induce clinical disease (Figure 1a). The IFN response to poly I:C was comparable to that observed following LCMV infection, as IFN- $\gamma$ levels were similar in mice 3 days after treatment or infection as measured by ELISA $(350 \mathrm{ng} / \mathrm{ml})$. To establish whether treatment was able to increase the level of insulitis without inducing diabetes, we stained pancreatic tissue sections with insulin-specific antibody to demonstrate any loss of insulin-containing $\beta$ cells. However, no increase in the level of peri-insulitis or insulitis was observed following treatment (Table 1). No histological changes were observed in the pancreas between age-matched treated and untreated BDC2.5 Tg mice (Figure 2a). To determine whether poly I:C treatment was able to activate lymphocytes in the BDC2.5 Tg mouse, lymphocytes were isolated from treated and untreated mice and stained with antibodies to cell surface molecules for analysis by flow cytometry. Although none of the $\mathrm{CD} 4^{+}$ $\mathrm{V}_{\beta} 4^{+}$(Tg TCR) lymphocytes showed changes in CD25, CD44, or CD69, indicating a lack of activation, the $\mathrm{CD} 4^{-} \mathrm{V}_{\beta} 4^{+} \mathrm{T}$ cell subset, representing primarily $\mathrm{CD}^{+} \mathrm{T}$ lymphocytes, demonstrated upregulation of these markers, denoting their activation (Figure 3a). Therefore, treatment of BDC2.5 Tg mice with poly I:C was comparable to infection with the noncytopathic virus, LCMV, and in neither case were the resting memory $\mathrm{Tg}$ $\mathrm{CD} 4^{+}$cells activated or disease induced.

Treatment with a single subdiabetogenic dose of STZ damages $\beta$ cells directly with only minimal immune cell involvement (14-17). While a multiple high- or low-dose regime of STZ leaves the mouse without functional $\beta$ cells and induces autoimmune diabetes, a single lowdose treatment causes significantly less damage and does not induce clinical disease $(14,15,17)$. During coxsackievirus infection of most mouse strains, limited damage to the $\beta$ cells is observed. The loss of a few $\beta$ cells is most likely counterbalanced by the limited ability of $\beta$ cells to regenerate. To mimic the minimal level of viral damage to the islet, mice would need to be treated with a single low dose of STZ that would damage only a few $\beta$ cells, leaving the majority of insulin-producing cells intact without altering clinical disease incidence on its own.

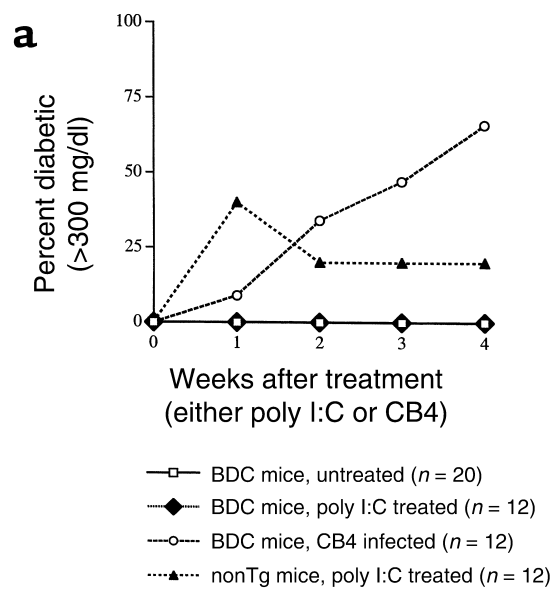

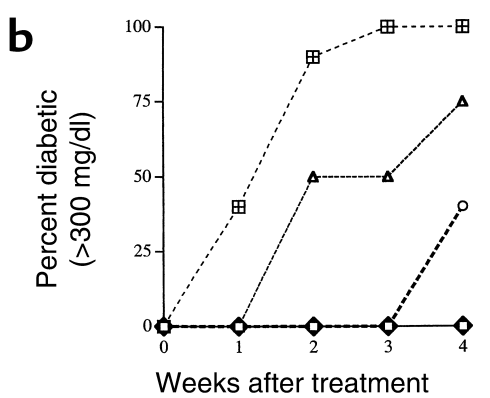

$$
\begin{aligned}
& \square \text { BDC mice, untreated }(n=20) \\
& - \text { BDC mice, buffer only }(n=4) \\
& \cdots-\text { BDC mice, STZ treated } 40 \mathrm{mg} / \mathrm{kg}(n=12) \\
& -\triangle \text { BDC mice, STZ treated } 80 \mathrm{mg} / \mathrm{kg}(n=12) \\
& \cdots \text { - BDC mice, STZ treated } 160 \mathrm{mg} / \mathrm{kg}(n=12)
\end{aligned}
$$

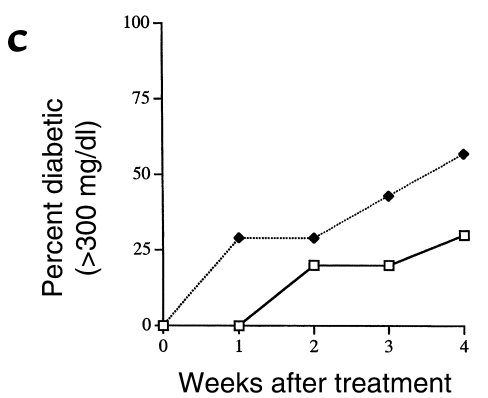

$\longrightarrow$ Untreated NODs $(n=10)$

-...... STZ treated NODs $(n=29)$

\section{Figure 1}

(a) Incidence of diabetes in BDC2.5 mice following poly I:C treatment. BDCTg mice and nontransgenic littermate controls were treated with $100 \mu \mathrm{g}$ of poly I:C. Alternatively, BDC Tg mice were infected with 100 pfu of CB4. Mice were monitored twice weekly for blood glucose, and mice with two consecutive values over $300 \mathrm{mg} / \mathrm{dl}$ were considered diabetic. Both BDC2.5 mice ( $n=12)$ and their nontransgenic littermates $(n=12)$ were analyzed and compared with untreated BDC Tg mice $(n=20)$. (b) Incidence of diabetes in BDC2.5 mice following STZ treatment. BDC Tg mice and nontransgenic littermates were treated with single doses of 0, 40, 80, or 160 mg/kg of STZ. Mice were monitored twice weekly for blood glucose, and mice with two consecutive values over $300 \mathrm{mg} / \mathrm{dl}$ were considered diabetic. (c) Incidence of diabetes in NOD/Shi mice following STZ treatment. Twelve-week-old NOD/Shi mice $(n=29)$ were treated with $80 \mathrm{mg} / \mathrm{kg}$ of STZ. Mice were monitored twice weekly for blood glucose, and mice with two consecutive values over $300 \mathrm{mg} / \mathrm{dl}$ were considered diabetic. Treated mice were analyzed and compared with untreated NOD/Shi mice $(n=10)$. Mice from all groups were age matched. Groups from $\mathbf{b}$ and $\mathbf{c}$ were compared for statistical significance by standard $\chi$-square tests. Of the relevant comparisons, significant differences were observed between STZ-treated $(80 \mathrm{mg} / \mathrm{kg})$ BDC mice and STZ-treated $(80 \mathrm{mg} / \mathrm{kg})$ NOD mice at both $2(P<0.001)$ and $4(P<0.001)$ weeks after treatment. 
Table 1

Summary of histological analysis of pancreatic sections from treated mice

\begin{tabular}{lccc}
\hline & Peri-insulitis & Insulitis & Total islets \\
Poly I:C - BDCs & $43 \%$ & $42 \%$ & 124 \\
STZ - BDCs & $7 \%$ & $85 \%$ & 114 \\
Untreated - BDCs & $42 \%$ & $56 \%$ & 113
\end{tabular}

Mice were sacrificed 4 weeks after treatment with poly I:C or STZ and examined for peri-insulitis and insulitis. Islets were scored for inflammatory cells as clean, peri-insulitis, or insulitis. Mice were matched for age and sex. Over 100 islets were scored per group. Scored groups were compared for statistical significance by standard $\chi$-square tests. Of the relevant comparisons, significant differences were observed for both peri-insulitis $(P<0.001)$ and insulitis $(P<0.001)$ between STZ-treated BDC mice and untreated BDC mice or poly I:C-treated BDC mice. Additionally, no significant difference was observed between poly I:C-treated and untreated BDC mice.

For this purpose, a pilot study was performed with a single treatment of STZ. One of three concentrations of $\mathrm{STZ}, 40,80$, or $160 \mathrm{mg} / \mathrm{kg}$, was administrated and mice were monitored for clinical disease. Initial treatments with STZ clearly indicated that induction of diabetes was dose-dependent (Figure 1b), and a single dose of 80 $\mathrm{mg} / \mathrm{kg}$ was chosen for further study. After administration of $80 \mathrm{mg} / \mathrm{kg}$ of STZ, BDC2.5 Tg mice developed IDDM in a manner and time frame similar to that induced by CB4 infection of BDC2.5 Tg mice (Figure $1 \mathrm{~b})$. That is, $67 \%$ of the mice became diabetic within $2-4$ weeks of treatment. To ensure that this was not an effect of the NOD MHC background, since this murine strain spontaneously develops diabetes, a large cohort of agematched (12-week-old) female NOD mice were treated simultaneously (Figure 1c). The NOD mice had a slight increase in the incidence of diabetes compared with that of spontaneous disease in our colony, but this incidence of STZ-induced NOD disease was significantly lower than that in the BDC2.5 Tg treated mice $(P<0.001)$.

STZ-treated BDC2.5 Tg mice had a marked loss of insulin-producing $\beta$ cells, which was accompanied by a dramatic rise in insulitis. The quantitated insulitis index showed a significant increase $(P<0.001)$ that coincided with the onset of diabetes (Table 1). To characterize the nature of these infiltrating lymphocytes, tissue sections were immunostained with antibody to CD4, CD25 (Figure $2 \mathrm{~b}$ ), and $\mathrm{V}_{\beta} 4$ (data not shown). The infiltrating cells were predominantly $\mathrm{CD} 4^{+}$lymphocytes and a sizable percentage of the infiltrating population was indeed $\mathrm{CD} 25^{+}$or IL-2 receptor-positive, reflecting an activated state (Figure 2b). As we had observed previously in the islets of CB4-infected mice (6), the predominant cell type was indeed the transgenic $\mathrm{T}$ cell and therefore both $\mathrm{CD}^{+}$and $\mathrm{V}_{\beta} 4^{+}$. Additionally, while loss of insulin staining in $\beta$ cells without strong insulitis is a common event in STZ-treated NOD mice (Figure 2a), this was not the case in the STZ-treated BDC mice since loss of insulin production by $\beta$ cells was closely associated with immune cell infiltration and insulitis. This outcome strongly suggests that resting memory $\mathrm{T}$ cells become activated due to the release of sequestered antigen during the environmental insult.
Local increases of activated macrophages in pancreatic islets accompany the progression of insulitis in mice developing diabetes $(22,23)$. This sequence is clearly visible in both NOD mice developing spontaneous diabetes and CB4-infected BDC Tg mice. Following single-dose STZ treatment of NOD mice, insulin production decreased without significant insulitis (Figure 2a). Consequently, fewer activated macrophages were present in the affected islets. To determine whether the islets of the STZ-treated BDC mice were phenotypically similar to the islets from virus-infected mice, pancreatic tissue sections were immunostained with $\mathrm{F} 4 / 80$ antibody to locate activated macrophages. As depicted in Figure $2 c$, the presence of macrophages in the islets of STZ-treated BDC mice closely resembled the situation in the islets of CB4infected BDC mice. In contrast, BDC mice treated with poly I:C and NOD mice given STZ did not exhibit pronounced insulitis, nor was there any significant increase in activated macrophages.

To correlate the shift in state from resting to activation in the transgenic $V_{\beta} 4^{+}$lymphocytes with the increase in insulitis and onset of diabetes, lymphocytes were isolated from the spleens and pancreatic lymph nodes of treated and untreated BDC2.5 mice and stained with antibodies to cell surface molecules including $\mathrm{V}_{\beta} 4$ (Tg TCR), IL-2 receptor (CD25), CD44, CD69, and L-selectin (CD62L) for analysis by flow cytometry. As previously reported, the predominant phenotype of the transgenic $\mathrm{CD} 4^{+} \mathrm{V}_{\beta} 4^{+}$population of $T$ cells signified resting memory as demonstrated by high expression of CD44 and absence of expression of CD25, CD69 (Figure 3b), and CD62L (data not shown). Strikingly, five days after STZ treatment, transgenic lymphocytes were activated, as confirmed by the increased numbers of $\mathrm{V}_{\beta} 4^{+}$lymphocytes staining for the activation markers CD25 and CD69, as well as the memory and activation marker CD44 (Figure 3). However, NOD mice had no obvious increase in activation in $\mathrm{CD}^{+} \mathrm{T}$ cells following STZ treatment. Additionally, similar STZ treatments of OT-II TCR Tg mice that express an MHC class II restricted TCR specific for ovalbumin (24) did not activate $T$ cells when measured by a similar FACS analysis for upregulation of the activation markers CD25, CD44, or CD69 (data not shown). Thus, the single low-dose treatment of STZ is acting solely as a $\beta$ cell toxin and did not directly activate $T$ cells. The shift in $T$ cell activation by a significant portion of $\mathrm{V}_{\beta} 4^{+}$cells correlated with the rapid and significant increase in insulitis composed predominantly of $\mathrm{V}_{\beta} 4^{+}(\mathrm{Tg})$ cells, $\beta$ cell loss, and observed diabetes similar to that observed following CB4 infection. Howev$\mathrm{er}$, in this case, a $\beta$ cell toxin replaced the virus, further underscoring the requirement for $\beta$ cell damage in the development of IDDM.

The ability of STZ to elicit diabetes with kinetics and phenotype similar to those of diabetes elicited by CB4 infection demonstrates that virus-mediated diabetes is the result of direct damage to the $\beta$ cells followed by 
activation of an autoreactive memory response that attacks and destroys the islets. The direct damage of $\beta$ cells associated with either the single low-dose STZ (at $80 \mathrm{mg} / \mathrm{kg}$ ) treatment or CB4 infection is, by itself, not sufficient to cause diabetes, since nontransgenic mice show little enhancement in disease. However, in both instances, sufficient $\beta$ cell damage occurs to allow for release of sequestered $\beta$ cell antigen and stimulation of the resting memory $\mathrm{T}$ cell population. This result, most likely, reflects the ability of pancreatic APCs to process and present $\beta$ cell antigen and thereby stimulate the BDC T cell.
To demonstrate that APCs take up islet antigen and stimulate the BDC $\mathrm{T}$ cells, we performed in vitro proliferation assays. APCs were enriched using plastic adherence from mice either treated or not treated with STZ; BDC T cells from untreated mice were then cocultivated with these APCs. As a control, APCs from untreated mice were cultured with BDC $\mathrm{T}$ cells and antigenic peptide (20). Previous work has demonstrated that enriched APCs can harbor antigen following viral infection or during local pathogenic inflammation and can present this antigen in in vitro proliferation assays $(19,25-27)$. In this situation, the pancreat- a

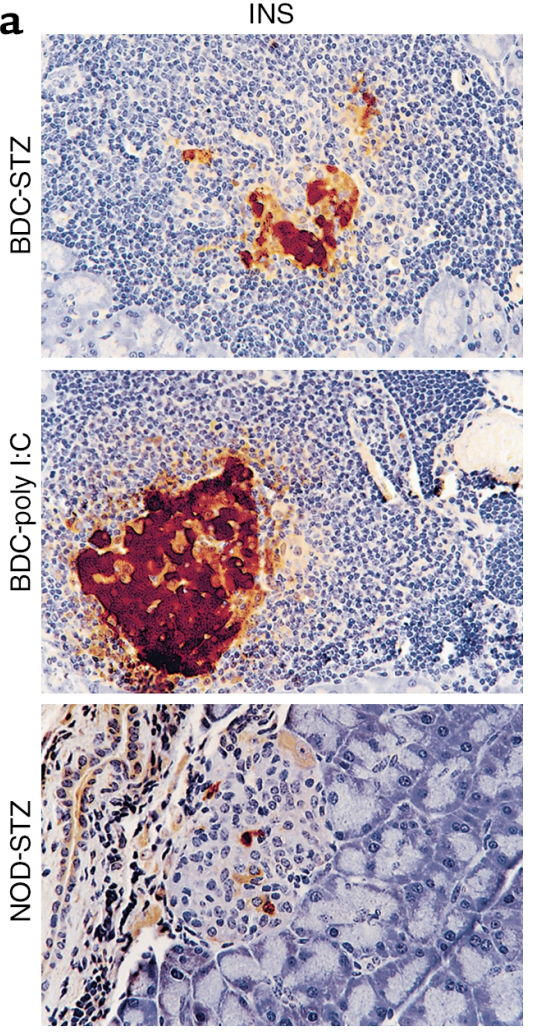

b

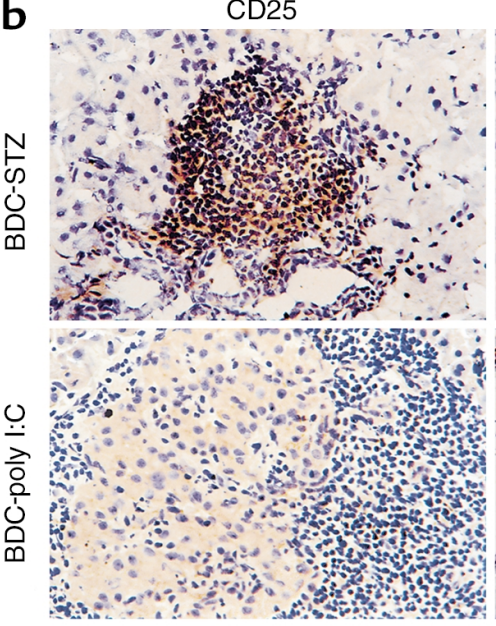

C
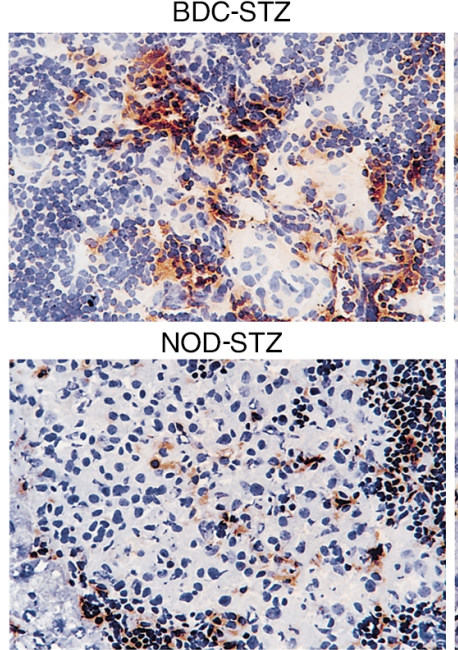

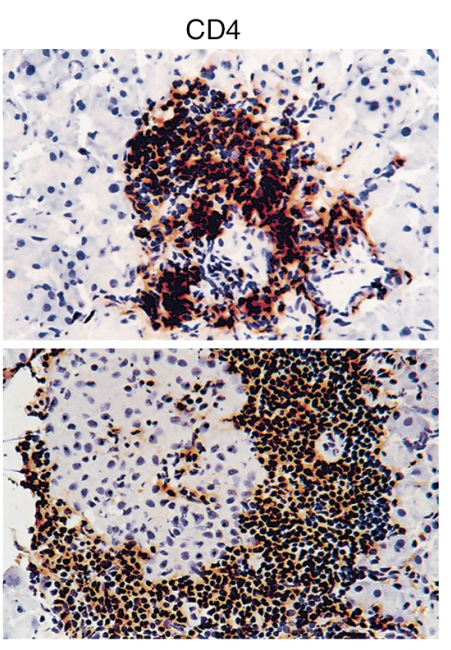

BDC-poly I:C

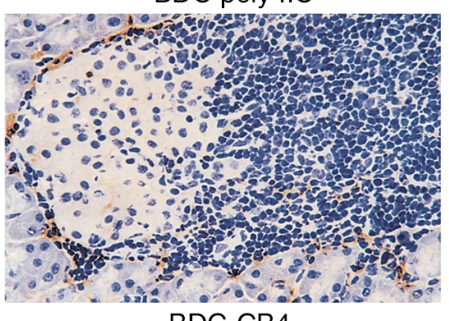

BDC-CB4

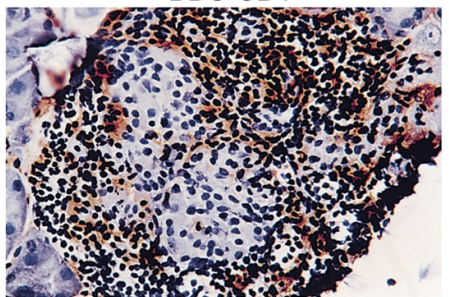

\section{Figure 2}

(a) Histological analysis of pancreata of treated BDC Tg and NOD mice. Representative sections of the pancreas from STZ-treated BDC Tg mice and poly I:C-treated BDC Tg mice reveal islet cell integrity and function. The function of $\beta$ cells was confirmed by immunohistochemistry using antibody to insulin (INS) ( $\times 400)$, present as brown staining. Mice were sacrificed at 2 weeks after treatment. (b) Immunohistological analysis of the islets from treated BDC Tg mice for activated T cells. Representative immunostained sections of islets from the pancreas of either STZ- or poly I:C-treated BDC Tg mice. Immunohistochemical staining for either CD4 or CD25 was performed and revealed CD4 $4^{+}$lymphocytes in both mice, but only STZ-treated mice have activated CD4+ cells as demonstrated by CD25 staining. Immunostained cells are brown (×400). The mice were sacrificed at 14 days after treatment. (c) Immunohistological analysis of pancreata from treated BDC $\mathrm{Tg}$ and NOD mice for macrophages. Representative immunostained sections of the pancreas from CB4-infected and STZ- and poly I:C-treated BDC Tg and NOD mice reveal differences in the number and activation state of resident macrophages. Antibody to the macrophage activation marker F4/80 was used to identify activated macrophages, which, following CB4 infection and STZ treatment of BDC Tg mice, appear to become activated in the peri-insular inflammation and to mobilize into the islets of Langerhans during insulitis $(\times 400)$. Brown antimacrophage staining is present over macrophages. Mice were sacrificed 2 weeks after infection or treatment. 

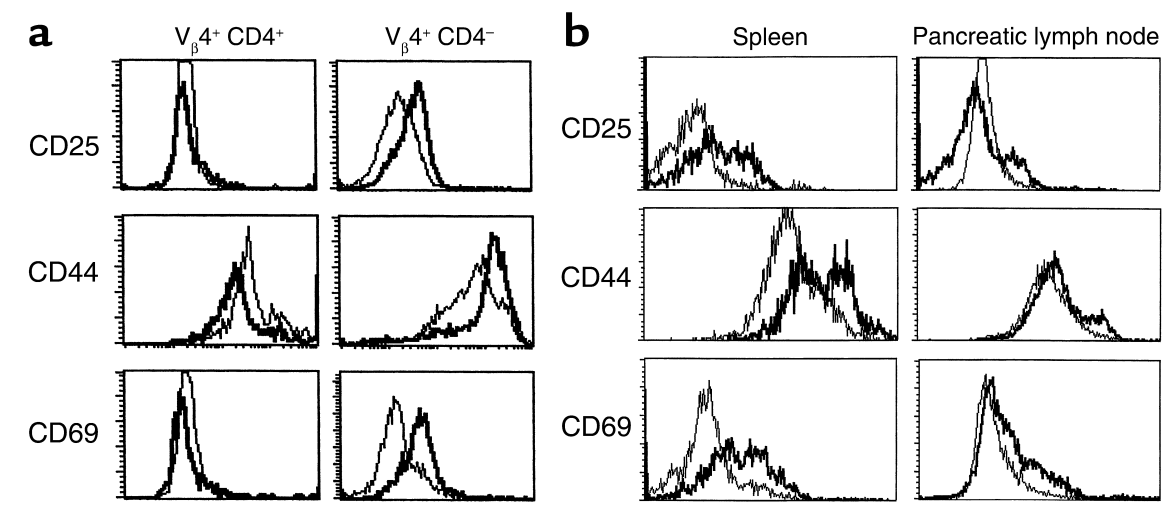

\section{Figure 3}

Analysis of the activation state of lymphocytes from BDC2.5 mice following treatment with either poly I:C (a) or STZ (b). Cells were stained with antibody to $V_{\beta} 4 T C R, C D 4$, and antibody to either CD25, CD44, or CD69. Cells were analyzed by FACS and compared to isotype controls. Shown are histograms of mean fluorescent intensities generated by gating on 10,000-20,000 CD $4^{+}, V_{\beta} 4^{+}$cells, or $V_{\beta} 4^{+}$, CD4- cells. (a). For both $\mathbf{a}$ and $\mathbf{b}$, the plots are representative of one of six mice analyzed, and all six had similar results. (a) Analysis of peripheral blood lymphocytes before (narrow) and three days following poly I:C treatment (wide) from identical mouse are displayed. No shift of activation is observed in the $\mathrm{V}_{\beta} 4^{+}, \mathrm{CD} 4^{+} \mathrm{T}$ cell population while activation is observed in the $\mathrm{V}_{\beta} 4^{+}, \mathrm{CD} 4^{-}\left(\mathrm{CD} 8^{+}\right) \mathrm{T}$ cell population posttreatment (PT). Changes in cell populations and gated markers $\mathrm{CD}^{+}, \mathrm{V}_{\beta} 4^{+}$: $\mathrm{CD} 25,5.7 \%$ to $7.2 \%$; CD44-high, $28.6 \%$ to $25.2 \%$; $\mathrm{CD} 69,6.7 \%$ to $6.9 \%$ and CD4- $\mathrm{V}_{\beta} 4^{+}$: CD25, $2.9 \%$ to $27.8 \%$; CD 44 -high, $22.9 \%$ to $65.0 \%$; CD69, $10.7 \%$ to $41.8 \%$. (b) Analysis of lymphocytes from the spleen and pancreatic lymph node before (narrow) and seven days following STZ treatment (wide) are displayed. Mice were analyzed at three, five, and seven days PT. Similar results were observed at five days, but not at three days. A dramatic shift in all three activation markers was observed in the spleen PT. A less dramatic shift was observed in the pancreatic lymph node.Changes in cell populations and gated markers in spleen $\mathrm{CD}^{+} \mathrm{V}_{\beta} 4^{+}$: $\mathrm{CD} 25,10.4 \%$ to $32.9 \%$; $\mathrm{CD} 44$-high, $19.8 \%$ to $56.5 \%$; $\mathrm{CD} 69,9.0 \%$ to $32.3 \%$; and in pancreatic lymph nodes, $\mathrm{CD}^{+} \mathrm{V}_{\beta} 4^{+}: \mathrm{CD}_{25}$, $5.4 \%$ to $25.3 \%$; CD 44 -high, $7.2 \%$ to $18.9 \%$; CD69, $8.7 \%$ to $27.0 \%$.

ic inflammation initially induced by the STZ treatment is minor in comparison to that reported in previous studies $(19,25-27)$, yet a subpopulation of APCs from these STZ-treated mice would be expected to have gathered endogenous islet cell antigen following STZinduced $\beta$ cell damage. These APCs would be capable of stimulating BDC $\mathrm{T}$ cells in culture to proliferate; however, stimulation would also induce these APCs to digest the harbored endogenous $\beta$ cell antigen rapidly (28). Additionally, unlike most standard in vitro proliferation assays in which antigen is not continuously available and is only brought into the culture within the APC. Therefore, the assay results should give lower stimulation indices than in the case of provided antigen or the peptide control. Additionally, the assay would peak in stimulation at a much earlier time point. In fact this was the case: the stimulation index peaked at day 3 of coculture and the index averaged about 4 for mice treated with STZ as compared with 1 for APCs from untreated mice (Figure 4). In the absence of exogenous antigen, APCs from mice with damaged $\beta$ cells were capable of stimulating the $\beta$ cell-specific BDC T cell, while APCs from mice without $\beta$ cell damage did not stimulate these $T$ cells. Therefore, following an insult to the pancreatic islets, the ability of the APC to process and present $\beta$ cell antigen to the BDC $\mathrm{T}$ cell is crucial to $\mathrm{T}$ cell activation.

To further demonstrate the importance of APC presentation and stimulation of $\mathrm{T}$ cells during the development of diabetes, adoptive transfer experiments were performed (Table 2). APCs enriched from STZtreated NODSCID mice induced diabetes when trans- ferred into naive BDC TCR Tg mice, while APCs from untreated mice did not induce disease. Conversely, to determine whether STZ treatment could prime mice for the development of disease, we transferred BDC2.5 $\mathrm{Tg} \mathrm{T}$ cells into STZ-treated recipients. The experiments showed that splenocytes from BDC TCR Tg mice induced rapid diabetes when transferred into STZtreated NODSCID mice. Splenocytes from BDC TCR $\mathrm{Tg}$ mice typically induce IDDM in NODSCID mice following transfer, but at a much slower rate and rarely before 4 weeks after transfer. Therefore the development of diabetes is the direct result of APCs presenting islet antigen from STZ-induced $\beta$ cell damage and activating BDC T cells.

In $\mathrm{BDC} \mathrm{Tg}$ mice, the autoreactive resting memory $\mathrm{T}$ cells are provided by transgenic technology. Development of this resting memory population of lymphocytes in prediabetic patients is, quite possibly, the consequence of previous pancreatropic infections. Whether given virus or STZ, the BDC Tg mice develop diabetes with similar kinetics, incidence, and severity. Taken together these data support the argument that diabetes is a result of environmental damage to the $\beta$ cells.

Alternatively, inflammation from environmental insults like CB 4 or STZ could stimulate the BDC TCR through a second TCR expressed with a different or nontransgenic $V_{\alpha}$ chain. The ability of the endogenous $\mathrm{V}_{\alpha}$ chain to avoid allelic exclusion and to recombine, be expressed, and interact with the transgenic $V_{\beta}$ chain has been well described (29-31). However, when these same BDC mice were crossed with another TCR Tg mouse to determine whether activation of the second 


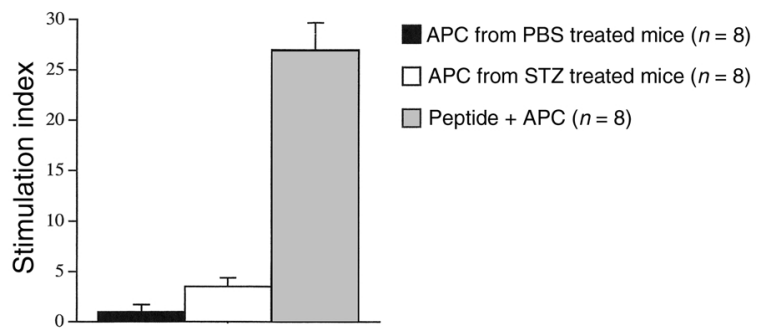

Figure 4

Presentation of endogenous antigen by APCs from STZ-treated mice activates $\beta$ cell-specific BDC T cells. Adherent mononuclear cells from NOD mice treated with STZ or PBS were prepared, irradiated, and cultured with BDC2.5 Tg splenocytes $\left(2 \times 10^{5}\right)$ at various ratios. Shown is a summary of results from the $4: 1$ ratio, $T$ cell $\left(2 \times 10^{5}\right)$ : APC $\left(5 \times 10^{4}\right)$. Also shown are the results of culturing these cells at $4: 1$ in the presence of antigenic peptide $(7 \mu \mathrm{M})(20)$ specific for the BDC T cell. Cultures were pulsed with $1 \mu \mathrm{Ci}$ of ${ }^{3} \mathrm{H}$ thymidine at 48 hours and harvested 18 hours thereafter. The data presented are from four separate experiments involving a total of eight mice in each group representing the APC populations. Values are presented as stimulation index + SEM as compared with BDC splenocytes incubated without additional APCs or antigen. Background in all experiments was between 500 and $2500 \mathrm{cpm}$.

TCR could activate BDC T cells, the results convincingly demonstrated that this could not occur in vivo to induce diabetes (32). One experimental drawback of this system is the lack of knowledge of the true BDCspecific antigen; however, recently, antigenic peptides have been identified that stimulate the BDC2.5 T (20). Although the nature of the natural islet autoantigen is not known, it is clear that an active response from $\mathrm{F} 4 / 80$-positive cells is required within the islet itself before disease develops. Activated macrophages or dendritic cells are most likely picking up released antigen and presenting it to resting memory $\mathrm{Tg} \mathrm{T}$ cells. Conversely, STZ-treated NOD mice do not have the same level of activated F4/80 staining cells and likewise exhibit less insulitis. Further, while APCs isolated from STZ-treated NOD or NODSCID mice successfully activated the BDC $\mathrm{T}$ cell and induced diabetes (Figure 4), they did not induce disease in NOD mice (data not shown). Therefore, mere stimulation of macrophages by STZ or $\beta$ cell damage does not neces- sarily activate $\mathrm{T}$ cells to induce disease. A critical level of autoreactive precursor resting memory $\mathrm{T}$ cells is most likely required $(33,34)$. Clearly, once initiated, the responses of both $\mathrm{T}$ cells and APCs amplify each other and heighten pathologic effects. The requirements of costimulation for this initiation are probably reduced, since the memory state of the $T$ cells lessens the priming requirements of these cells.

NOD mice treated with STZ did not exhibit an increase in diabetes development despite the release of islet antigen and the prevalence of autoreactive lymphocytes. The major difference between the BDC mice, in which diabetes is STZ-inducible, and the NOD mice is the frequency of autoreactive precursor resting memory $\mathrm{T}$ cells. The transgenic population consists primarily of $\mathrm{CD}^{+}$, CD44-high, CD62L-low islet-specific lymphocytes with few requirements for activation. In the NOD mouse, these autoreactive lymphocytes are present at a much lower frequency. Despite the predominance of a single specific $T$ cell population, the BDC Tg mouse had enough flexibility to combat viral infections and counter-regulate its large number of autoreactive cells. This abnormal $\mathrm{T}$ cell repertoire of the BDC mouse is obviously an exaggerated version of a human preclinical situation.

In patients, genetic predisposition and environmental exposures could lead to a similar expansion of autoreactive $T$ cells, leading to peri-insulitis. This repertoire could undergo further distortion in response to viral infection, as has been reported by others (35-37). Ultimately, release of sequestered antigen by viral infection or environmental insult would be the triggering event in disease initiation, as observed in the STZ-treated or CB4-infected BDC mouse. It was previously proposed that, in a situation with a distorted lymphocyte repertoire, infection could shift the balance and result in a loss of regulatory $\mathrm{T}$ cells, allowing disease to occur. Similarly, viral interference of the host immune regulatory process has been observed in mice with normal $T$ cell repertoires, and the result was an increased survival rate of nonviral-specific T cells $(11,38,39)$. However, a single low dose of STZ (acting in place of CB4) did not directly activate $T$ cells or interfere with the turnover or regulation of autoreactive cells. STZ can

Table 2

Transfer of autoimmune diabetes with APCs from mice with damaged $\beta$ cells

Donor cells Recipient mice Weeks post-transfer (\% diabetic)

APCs from untreated NODSCID mice

APCs from STZ-treated NODSCID mice (7 days PT)

BDC2.5 TCR Tg splenocytes

BDC2.5 TCR Tg splenocytes

$\begin{array}{lcccccc}\text { BDC2.5 TCR } \operatorname{Tg}(n=9)^{\mathrm{A}} & 0 \% & 0 \% & 0 \% & 0 \% & 0 \% & 0 \% \\ \text { BDC2.5 TCR } \operatorname{Tg}(n=9)^{\mathrm{A}} & 11 \% & 33 \% & 33 \% & 33 \% & 33 \% & 33 \% \\ \text { Untreated NODSCID mice }(n=9)^{\mathrm{A}} & 0 \% & 0 \% & 0 \% & 44 \% & 44 \% & 55 \% \\ \text { STZ-treated NODSCID mice } & 60 \% & 70 \% & 70 \% & 80 \% & 90 \% & 90 \% \\ (7 \text { days PT, } n=10)^{\mathrm{A}} & & & & & & \end{array}$

Autoimmune diabetes was induced following either adoptive transfer of APCs from mice with damaged $\beta$ cells or transfer of autoreactive T cells into immunedeficient mice with damaged $\beta$ cells. Adherent mononuclear cells from NODSCID mice treated with STZ or not treated were adoptively transferred into BDC2.5 $\mathrm{Tg}$ mice at a concentration of $5 \times 10^{5}$ to $10 \times 10^{5}$ cells per mouse. Splenocytes from BDC2.5 TCR Tg mice were adoptively transferred into NODSCID mice that had been STZ-treated or not 7 days previously at a concentration of $2 \times 10^{6}$ to $5 \times 10^{6}$ cells per mouse. Mice with consecutive BG measurements of greater than $300 \mathrm{mg} / \mathrm{dl}$ were considered diabetic. ${ }^{A}$ Age-matched. PT, post-transfer. 
fully replace the virus in IDDM development; thus the role of CB 4 is clearly to damage $\beta$ cells and not to act as an immune modulator.

The inability of poly I:C to upregulate the BDC $\mathrm{Tg} T$ cell and initiate disease argues against a role for bystander $\mathrm{T}$ cell amplification in the development of virus-mediated autoimmune disease. Although viruses have the ability to upregulate nonspecific responses and to enhance disease, this bystander amplification is subject to limitations in the scope of its involvement. Results from other investigators $(40,41)$ imply that an additional factor is required to reactivate lymphocytes and drive autoimmunity. Merely activating potential autoimmune $T$ cells is not enough to cause disease. Yet, in some instances, viral cross-activation has increased the incidence of disease $(42,43)$. Crossactivation during infection is predominantly associated with $\mathrm{CD}^{+} \mathrm{T}$ cells through the type 1 IFN pathway and involves IL- 15 and the use of the IL- $2 \beta$ receptor that is specifically found on $\mathrm{CD}^{+}$, but not $\mathrm{CD} 4^{+}, \mathrm{T}$ cells. Conversely, $\mathrm{CD} 4^{+} \mathrm{T}$ cells are not similarly reactivated. This activation of $\mathrm{CD}^{+} \mathrm{T}$ cells by IFN was induced by IL-15 and appeared to utilize the IL-2 receptor $\beta$ that is found on $\mathrm{CD}^{+} \mathrm{T}$ cells but not $\mathrm{CD}^{+}$ $\mathrm{T}$ cells (44). Although $\mathrm{CD}^{+} \mathrm{T}$ cells participate in nonspecific cross-activation, systems involving $\mathrm{CD}^{+}{ }^{+} \mathrm{ym}-$ phocytes, as in coxsackievirus-induced IDDM of BDC2.5 Tg mice (6), herpes simplex virus-1-induced herpetic stromal keratitis (45), and pathogen-induced experimental autoimmune encephalomyelitis (46), show little cross-activation. In systems dependent on $\mathrm{CD}^{+}$lymphocytes, such as the LCMV transgenic models $(43,47)$, systemic cross-activation may be the only requirement for activating memory $\mathrm{CD} 8^{+} \mathrm{T}$ cells, inducing pathogenic $\mathrm{T}$ cells and producing disease. Additionally, in general, $\mathrm{CD}^{+} \mathrm{T}$ cell effectors have fewer counter-regulatory mechanisms than do $\mathrm{CD} 4^{+}$ $T$ cells. In the case of human autoimmune disease, the actions of $\mathrm{CD}^{+} \mathrm{T}$ cells may, in turn, be more important during relapses and be critical to the development of both IDDM and multiple sclerosis. Certainly $\mathrm{CD}^{+}$ and $\mathrm{CD}^{+} \mathrm{T}$ cells are both important participants in human autoimmune disease. Their reactivation, apparently via separate mechanisms, is clearly mutually dependent. Differences in the inducibility of diabetes between the BDC2.5 Tg mouse and its NOD littermate further elucidate not only the crucial importance of breakdown and release of sequestered antigen, but also the requirement for a sizable preexisting memory population of autoreactive lymphocytes in the development of overt clinical disease.

\section{Acknowledgments}

We would like to thank Shyam Pakala, Malin Flodstrom, Cecile King, and Michelle Krakowski for many helpful discussions. M.S. Horwitz was a recipient of an American Diabetes Association Career Development Award. N. Sarvetnick was supported by a Diabetes Interdisciplinary Research Program grant from the
Juvenile Diabetes Foundation International and grant 1RO1 AI42231 from the National Institute of Allergy and Infectious Diseases. This is manuscript no. 13276IMM from The Scripps Research Institute.

1. Andreoletti, L., et al. 1997. Detection of coxsackie B virus RNA sequences in whole blood samples from adult patients at the onset of type I diabetes mellitus. J. Med. Virol. 52:121-127.

2. Hyoty, H., et al. 1995. A prospective study of the role of coxsackie B and other enterovirus infections in the pathogenesis of IDDM. Childhood Diabetes in Finland (DiMe) Study Group. Diabetes. 44:652-657.

3. Webb, S., Loria, R., Madge, G., and Kibrick, S. 1976. Susceptibility of mice to group B coxsackie virus is influenced by the diabetic gene. J. Exp. Med. 143:1239-1248.

4. Yoon, J., Onodera, T., and Notkins, A. 1978. Virus-induced diabetes mellitus. XV. Beta cell damage and insulin-dependent hyperglycemia in mice infected with coxsackie virus B4. J. Exp. Med. 148:1068-1080.

5. Atkinson, M., et al. 1994. Cellular immunity to a determinant common to glutamate decarboxylase and coxsackie virus in insulin-dependent diabetes. J. Clin. Invest. 94:2125-2129.

6. Horwitz, M.S., et al. 1998. Coxsackie virus-induced diabetes: initiation by bystander damage and not molecular mimicry. Nat. Med. 4:781-785.

7. Katz, J.D., Wang, B., Haskins, K., Benoist, C., and Mathis, D. 1993. Following a diabetogenic $\mathrm{T}$ cell from genesis through pathogenesis. Cell. 74:1089-1100.

8. Dyrberg, T., Schwimmbeck, P.L., and Oldstone, M.B. 1988. Inhibition of diabetes in BB rats by virus infection. J. Clin. Invest. 81:928-931.

9. Oldstone, M.B. 1988. Prevention of type I diabetes in nonobese diabetic mice by virus infection. Science. 239:500-502.

10. Butz, E.A., and Bevan, M.J. 1998. Massive expansion of antigen-specific CD8 T cells during acute virus infection. Immunity. 8:167-175.

11. Mitchell, T., Kappler, J., and Marrack, P. 1999. Bystander virus infection prolongs activated T cell survival. J. Immunol. 162:4527-4535.

12. Murali-Krishna, K., et al. 1998. Counting antigen-specific CD8 T cells: a reevaluation of bystander activation during viral infection. Immunity. 8: $177-187$.

13. Tough, D., Borrow, P., and Sprent, J. 1996. Induction of bystander T cell proliferation by viruses and type I interferon in vivo. Science. 272:1947-1950.

14. Wilson, G.L., and Leiter, E.H. 1990. Streptozotocin interactions with pancreatic $\beta$ cells and the induction of insulin-dependent diabetes. Curr. Top. Microbiol. Immunol. 156:27-54.

15. Ito, M., Kondo, Y., Nakatani, A., and Naruse, A. 1999. New model of progressive non-insulin-dependent diabetes mellitus in mice induced by streptozotocin. Biol. Pharm. Bull. 22:988-989.

16. Gerling, I.C., Friedman, H., Greiner, D.L., Shultz, L.D., and Leiter, E.H. 1994. Multiple low-dose streptozocin-induced diabetes in NOD-scid/scid mice in the absence of functional lymphocytes. Diabetes. 43:433-440.

17. Ihm, S., Lee, K., McArthur, R., and Yoon, J. 1990. Predisposing effect of anti-beta cell autoimmune process in NOD mice on the induction of diabetes by environmental insults. Diabetologia. 33:709-712.

18. Horwitz, M.S., Krahl, T., Fine, C., Lee, J., and Sarvetnick, N. 1999. Protection from lethal coxsackievirus-induced pancreatitis by expression of gamma interferon. J. Virol. 73:1756-1766.

19. King, C., et al. 2001. Interleukin-4 acts at the locus of the antigen presenting dendritic cell to counter regulate cytotoxic CD8+ T cell responses. Nat. Med. 7:206-214.

20. Judkowski, V., et al. 2001. Identification of MHC class II-restricted peptide ligands, including a glutamic acid decarboxylase 65 sequence, that stimulate diabetogenic T cells from transgenic BDC2.5 nonobese diabetic mice. J. Immunol. 166:908-917.

21. Tough, D.F., and Sprent, J. 1996. Viruses and T cell turnover: evidence for bystander proliferation. Immunol. Rev. 150:129-142.

22. Hutchings, P., et al. 1990. Transfer of diabetes in mice prevented by blockade of adhesion-promoting receptor on macrophages. Nature. 348:639-642.

23. Jun, H.S., Yoon, C.S., Zbytnuik, L., van Rooijen, N., and Yoon, J.W. 1999. The role of macrophages in T cell-mediated autoimmune diabetes in nonobese diabetic mice. J. Exp. Med. 189:347-358.

24. Barnden, M.J., Allison, J., Heath, W.R., and Carbone, F.R. 1998. Defective TCR expression in transgenic mice constructed using cDNA $\alpha$ and $\beta$ chain genes under the control of heterologous regulatory elements. Immunol. Cell Biol. 76:34-40.

25. Katz-Levy, Y., et al. 1999. Endogenous presentation of self myelin epitopes by CNS-resident APCs in Theiler's virus-infected mice. J. Clin. Invest. 104:599-610.

26. Green, E., Eynon, E., and Flavell, R. 1998. Local expression of TNFalpha in neonatal NOD mice promotes diabetes by enhancing presentation of islet antigens. Immunity. 9:733-743.

27. Carson, M.J., Sutcliffe, J.G., and Campbell, I.L. 1999. Microglia stimu- 
late naive $\mathrm{T}$ cell differentiation without stimulating $\mathrm{T}$ cell proliferation. J. Neurosci. Res. 55:127-134.

28. Turley, S.J., et al. 2000. Transport of peptide-MHC class II complexes in developing dendritic cells. Science. 288:522-527.

29. Padovan, E., et al. 1993. Expression of two T cell receptor alpha chains: dual receptor T cells. Science. 262:422-424.

30. Heath, W., et al. 1995. Expression of two T cell receptor alpha chains on the surface of normal murine T cells. Eur. J. Immunol. 25:1617-1623.

31. Simpson, E., et al. 1995. T cells with dual antigen specificity in T cell receptor transgenic mice rejecting allografts. Eur. J. Immunol. 25:2813-2817.

32. Fossati, G., Cooke, A., Papafio, R.Q., Haskins, K., and Stockinger, B. 1999. Triggering a second T cell receptor on diabetogenic T cells can prevent induction of diabetes. J. Exp. Med. 190:577-583.

33. Horwitz, M.S., Fine, C., Ilic, A., and Sarvetnick, N. 2001. Requirements for viral-mediated autoimmune diabetes: $\beta$ cell damage and immune infiltration. J. Autoimmun. 16:211-217.

34. Serreze, D.V., Ottendorfer, E.W., Ellis, T.M., Gauntt, C.J., and Atkinson, M.A. 2000. Acceleration of type 1 diabetes by a coxsackievirus infection requires a preexisting critical mass of autoreactive $T$ cells in pancreatic islets. Diabetes. 49:708-711.

35. Kalams, S., et al. 1994. Longitudinal analysis of T cell receptor (TCR) gene usage by human immunodeficiency virus 1 envelope-specific cytotoxic T lymphocyte clones reveals a limited TCR repertoire. J. Exp. Med. 179:1261-1271.

36. Pantaleo, G., et al. 1994. Major expansion of CD8+ T cells with a predominant $\mathrm{V}$ beta usage during the primary immune response to HIV. Nature. 370:463-467.

37. Prevost-Blondel, A., et al. 1997. In vivo longitudinal analysis of a dominant TCR repertoire selected in human response to influenza virus.
Virology. 233:93-104.

38. Selin, L., et al. 1999. Attrition of T cell memory: selective loss of LCMV epitope-specific memory CD8 $\mathrm{T}$ cells following infections with heterologous viruses. Immunity. 11:733-742.

39. Selin, L., Varga, S., Wong, I., and Welsh, R. 1998. Protective heterologous antiviral immunity and enhanced immunopathogenesis mediated by memory T cell populations. J. Exp. Med. 188:1705-1715.

40. Zarozinski, C.C., and Welsh, R.M. 1997. Minimal bystander activation of CD8 T cells during the virus-induced polyclonal T cell response. J. Exp. Med. 185:1629-1639.

41.Zhao, Z.-S., Granucci, F., Yeh, L., Schaffer, P.A., and Cantor, H. 1998. Molecular mimicry by herpes simplex virus-type 1: autoimmune disease after viral infection. Science. 279:1344-1347.

42. Miller, S.D., et al. 1997. Persistent infection with Theiler's virus leads to CNS autoimmunity via epitope spreading. Nat. Med. 3:1-4.

43. Evans, C., Horwitz, M., Hobbs, M., and Oldstone, M. 1996. Viral infection of transgenic mice expressing a viral protein in oligodendrocytes leads to chronic central nervous system autoimmune disease. J. Exp. Med. 184:2371-2384.

44. Von Herrath, M.G., Dockter, J., and Oldstone, M.B.A. 1994. How virus induces a rapid or slow onset insulin-dependent diabetes mellitus in a transgenic model. Immunity. 1:231-242.

45. Gangappa, S., Babu, J., Thomas, J., Daheshia, M., and Rouse, B. 1998. Virus-induced immunoinflammatory lesions in the absence of viral antigen recognition. J. Immunol. 161:4289-4300.

46. Hafler, D., Fos, D., Benjamin, M., Blue, M., and Weiner, H. 1987. Secondary immune amplification following live poliovirus immunization in humans. Clin. Immunol. Immunopathol. 44:321-328.

47. Oldstone, M. 1987. Molecular mimicry and autoimmune disease. Cell. 50:819-820. 